\title{
RF PROPAGATION MEASUREMENT AND MODEL VALIDATION DURING RF/IR SYNERGY TRIAL VAMPIRA
}

\author{
Eric Heemskerk \\ TNO Defence, Security and Safety, Department of Observation Systems \\ P.O. Box 96864, 2509 JG The Hague, The Netherlands
}

\begin{abstract}
The member nations of AC/323 SET-RTG056/RTG32 on Integration of Radar and Infrared for Ship Self Defence have performed the Validation Measurements for Propagation in the Infrared and Radar (VAMPIRA). The objective was to get insight into the radar and infrared synergy concentrated on propagation in a coastal environment including horizontal inhomogeneity and to validate radar and infrared propagation models. The trial was held in the period 25 March-5 April 2004 near Surendorf Germany. As part of the trial TNO made RF 1-way transmission measurements, 24 hours/day during the whole trial period. The transmission path over the Eckernförder Bucht in Northern Germany had a length of $8.2 \mathrm{~km}$. The transmitted signal was a sweep consisting of 6 frequencies i.e. 3.36, 5.32, 8.015, 9.7, 13.45, and $15.71 \mathrm{GHz}$. The transmitter height was $11.5 \mathrm{~m}$, the receiver height $6.4 \mathrm{~m}$ above 'normal null'. At each end of the path a meteorological station was installed measuring every 30 s the air temperature, relative humidity, air pressure, wind speed and wind direction. About halfway the path the TNO meteo buoy was anchored measuring air temperature and relative humidity at 5 heights between 0.65 and $5.15 \mathrm{~m}$ above the sea surface. Also the sea water temperature was measured by the buoy on a depth of $1 \mathrm{~m}$ below the sea surface. The effects of evaporation ducting at the propagation at the various frequencies were clearly demonstrated. Some times very deep fadings were present at 13.45 and $15.71 \mathrm{GHz}$ where at the same time almost no effect at 3.36 and $5.32 \mathrm{GHz}$ was observed. The measured propagation at $15.71 \mathrm{GHz}$ was more enhanced than at $13.45 \mathrm{GHz}$ due to the ducting conditions and the elevation angle of the transmitter and receiver antenna. In several sample cases the 1-way propagation factors are computed for every 5 minutes using the propagation model TERPEM (Signal Science) and the vertical refractivity profiles computed by the TNO model TARMOS. The 1way computed propagation factors compared very well to the measured data at all frequencies, although the computed fadings were not always as deep as the measured ones. A first promising result has been obtained computing the observed height of the RF source under various atmospheric conditions using the transmission phases computed by TERPEM.
\end{abstract}

Keywords: electromagnetic propagation, model validation, measurement, radar, atmospheric refraction, ducting

\section{INTRODUCTION}

In the last decade many operations of the navies have been moved from open ocean towards littoral/warm waters. Operating in littoral environment includes new threats. Also the background and propagation under atmospheric conditions with a strong horizontal inhomogeneity have a severe impact on the radar and infrared sensor performance, on the detection / classification / recognition of small targets in the coastal region and on the ship self defence. Individual onboard sensors are all differently affected by the environment, depending on height, frequency, bearing, range etc. Simultaneous exploitation of radar and infrared sensors and multi-sensor fusion can overcome the difficulties with respect to target detection/recognition/classification by the individual sensors in a coastal environment.

Within this context the so-called radar-infrared synergy with respect to propagation through the marine boundary layer plays an important role. The simultaneous occurrence of RF and IR sub- and superrefractive conditions is a part of the synergy and complementarity of the RF and IR wavelengths for detection of targets.

The member nations of NATO AC/323 SET-056/RTG32 are investigating this synergy. The first part of the RTG study included a review of existing data. It revealed that there was only one case where three from the four possible combinations of radar and infrared sub- and superrefractive conditions occurred. But the trial only contained radar and

Optics in Atmospheric Propagation and Adaptive Systems VIII, edited by Karin Stein, Anton Kohnle

Proceedings of SPIE Vol. 5981 (SPIE, Bellingham, WA, 2005)

0277-786X/05/\$15 - doi: 10.1117/12.637616

Proc. of SPIE Vol. $5981598107-1$ 
environmental measurements. Therefore the RTG members organised the Validation Measurements for Propagation in the Infrared and Radar (VAMPIRA) to obtain sufficient and appropriate experimental data on simultaneous radar and infrared propagation [1].

\section{VAMPIRA TRIAL}

VAMPIRA was held from 25 March-5 April 2004 near Surendorf (Schwedeneck) Germany. The location and time period were selected from the results of a study on the refractive conditions during previous trials and using a 5-year meteorological database of the Surendorf area. The trial area with an overview of instrumentation and assets is given in figure 1.

The objectives of VAMPIRA were to obtain a complete data set of simultaneously measured environmental parameters, and radar and infrared propagation factors to get insight into the radar and infrared synergy concentrated on propagation in a coastal environment including horizontal inhomogeneity and to validate radar and infrared propagation models.

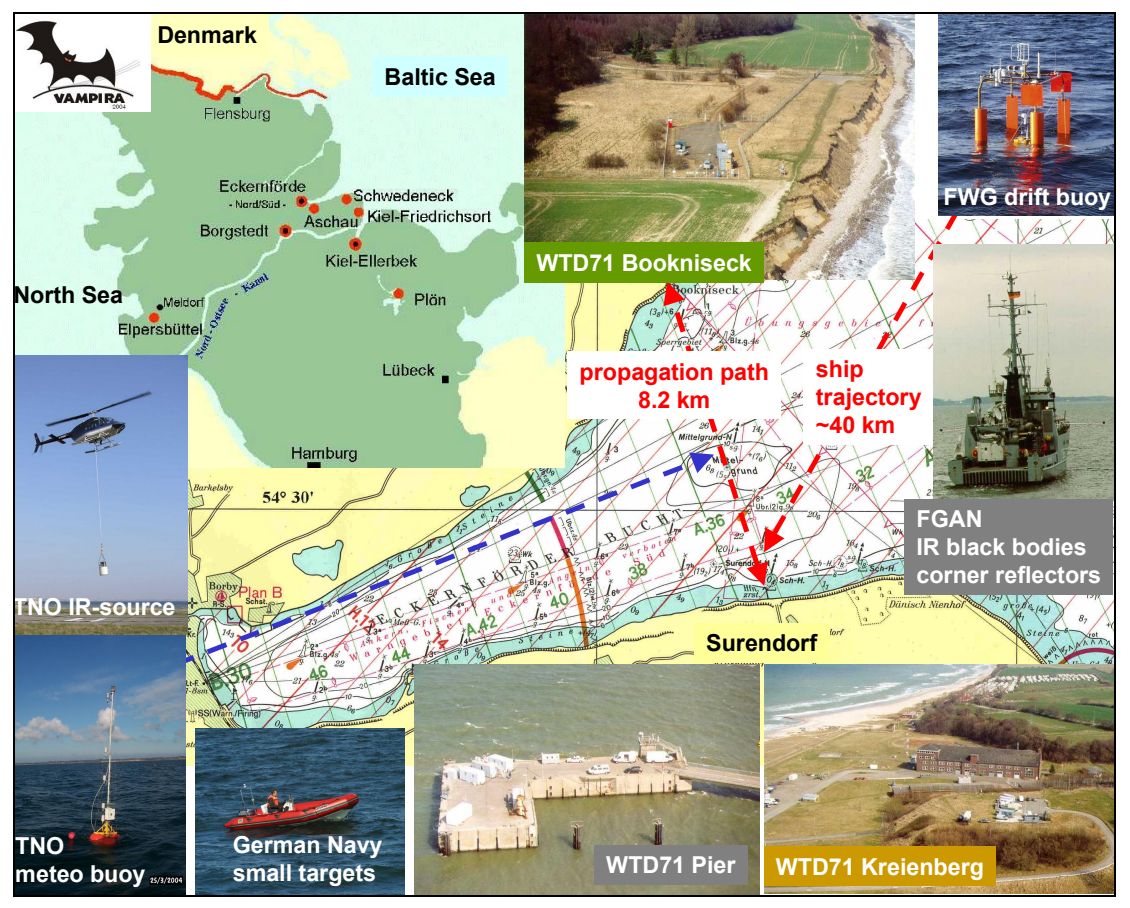

Figure 1: VAMPIRA trial area, meteo buoys and assets.

The measurements included

- $\quad$ simultaneous 1-way radar and infrared transmission measurements over a $8.2 \mathrm{~km}$ path between Surendorf and Bookniseck 24 hours/day during the whole trial period,

- simultaneous radar and EO measurements on radar corner reflectors respectively black bodies and visible lights on the ship Stollergrund during inbound and outbound runs (path length max $40 \mathrm{~km}$ ) at various times of the day,

- simultaneous radar and EO measurements on an IR source suspended under a helicopter at various times of the day,

- simultaneous radar and EO measurements on small targets,

- meteorological measurements on the pier and Kreienberg in Surendorf (24 hours/day),

- meteorological measurements at Bookniseck (24 hours/day),

- meteorological measurements by a buoy about halfway Surendorf-Bookniseck (24 hours/day),

- $\quad$ environmental measurements by 2 free drifting buoys near the ship trajectory during the ship runs ( $>8$ hours/day),

- radiosoundings from the pier and from the ship Helmsand (once per hour during the ship runs),

- $\quad$ 1-way RF propagation measurement with a receiver on the Stollergrund. 
This paper only deals with the RF 1-way propagation measurements between Surendorf and Bookniseck including the corresponding meteo. First results have been presented in [2].

For the 1-way RF propagation measurements the transmitter was positioned at Bookniseck, the receiver on the pier on the WTD71 (Wehrtechnische Dienststelle71) facility at Surendorf (Schwedeneck) Germany. The height of the transmitter and receiver antenna was 11.5 and $6.4 \mathrm{~m}$ above 'normal null' respectively. The 1-way RF propagation measurements were made by TNO once per minute during the trial period. The path length was $8.2 \mathrm{~km}$. The transmitted signal included a sweep over 6 frequencies i.e. 3.36, 5.32, 8.015, 9.7, 13.45, and $15.71 \mathrm{GHz}$. Each frequency was transmitted during $9 \mathrm{~s}$. The sweep repetition time was $60 \mathrm{~s}$. At each end of the path a meteorological station was installed measuring every $30 \mathrm{~s}$ the air temperature, relative humidity, air pressure, wind speed and wind direction. About halfway the path the TNO meteo buoy was anchored. This buoy measured the air temperature and relative humidity at 5 heights from $0.5-5 \mathrm{~m}$ above the sea level once per minute, but the data was averaged over 5 minutes. Also the sea water temperature was measured by the buoy at a depth of $1 \mathrm{~m}$.

\section{METEOROLOGICAL AND DUCTING CONDITIONS ALONG THE PATH}

\subsection{Meteorological conditions}

The meteo buoy was positioned about halfway the path, which is a good position for obtaining representative data of the meteorological conditions along the transmission path. Land effects did affect temperature and humidity data that were collected at each end of the path. For instance the air temperature measured at the pier was on average about $2{ }^{\circ} \mathrm{C}$ higher than the one measured by the buoy. Of course this depends on the wind direction. The data from the buoy sensor at 5.15 $\mathrm{m}$ was selected for use in the analysis. Since the buoy did not contain an anemometer the meteo data set was completed by the wind speed measured at the pier. This was allowed because a comparison of the wind speeds measured at the Kreienberg (in Surendorf), the pier and Bookniseck showed only small differences. Figure 2 gives the measured air and sea temperature and the relative humidity from the buoy and the wind speed and wind direction from the pier during the whole trial period.

The sea temperature showed only small variations during the trial period. The air temperature however varied largely between $0^{\circ}$ and $8^{\circ}$, resulting in positive as well as negative ASTD values during the trial. These correspond to stable and unstable conditions respectively. Also the relative humidity showed large variations. In the first half of the trial a few rain events took place after a few days with moderate values of humidity. At the end of the trial a few time periods occurred with a sudden drop of humidity and a simultaneous rise respectively drop of the air temperature that might have given rise to radar subrefractive conditions as will be discussed later on.

The wind was blowing from the open sea during about $24 \%$ of the time. During about $20 \%$ of the trial the wind was coming from directions between $90^{\circ}$ and $120^{\circ}$ i.e. partly from open sea but affected by the land. The remaining of the time the wind was blowing from the land. Most of the time the wind direction was eastern. During $37 \%$ of the time the wind direction varied between $180^{\circ}$ and $240^{\circ}$. Only during $8 \%$ of the time there was western to northern wind.

The wind speed varied strongly during the trial between 1 and $10 \mathrm{~m} / \mathrm{s}$. It means that based upon the observations made near Surendorf and Bookniseck the atmospheric conditions changed quite a lot during the trial including land effects as well during approximately half the trial period. A detailed description of the atmospheric conditions based upon buoy measurements in open sea at a range beyond $10 \mathrm{~km}$ from Surendorf is given in [3]. The latter study indicates that there might have been rather strong horizontally inhomogeneous conditions during the trial period all over the trial area towards northern directions i.e. along the ship runs.

At the beginning of the trial period the ASTD was negative, at the end of the trial positive ASTD values were met. Especially in the first part of the trial the relative humidity showed large variations over the day $(60-100 \%)$. Noteworthy is the $3^{\text {rd }}$ April where around 06:00 UTC the air temperature increased within a few hours from 4 to $8^{\circ} \mathrm{C}$, while the relative humidity dropped from $80 \%$ to $60 \%$ within an hour and increased within the next 5 hours till about $90 \%$. A comparable phenomenon occurred to a less extent also on $4^{\text {th }}$ April around 11:00 UTC. Both phenomena have been observed also by the meteo station on the pier, at the Kreienberg and in Bookniseck. On the $4^{\text {th }}$ April the sudden temperature and humidity decreases coincide with an also sudden change in wind direction from south (coming from the land) towards $240^{\circ}$ (coming almost along the Eckernförder Bucht). 


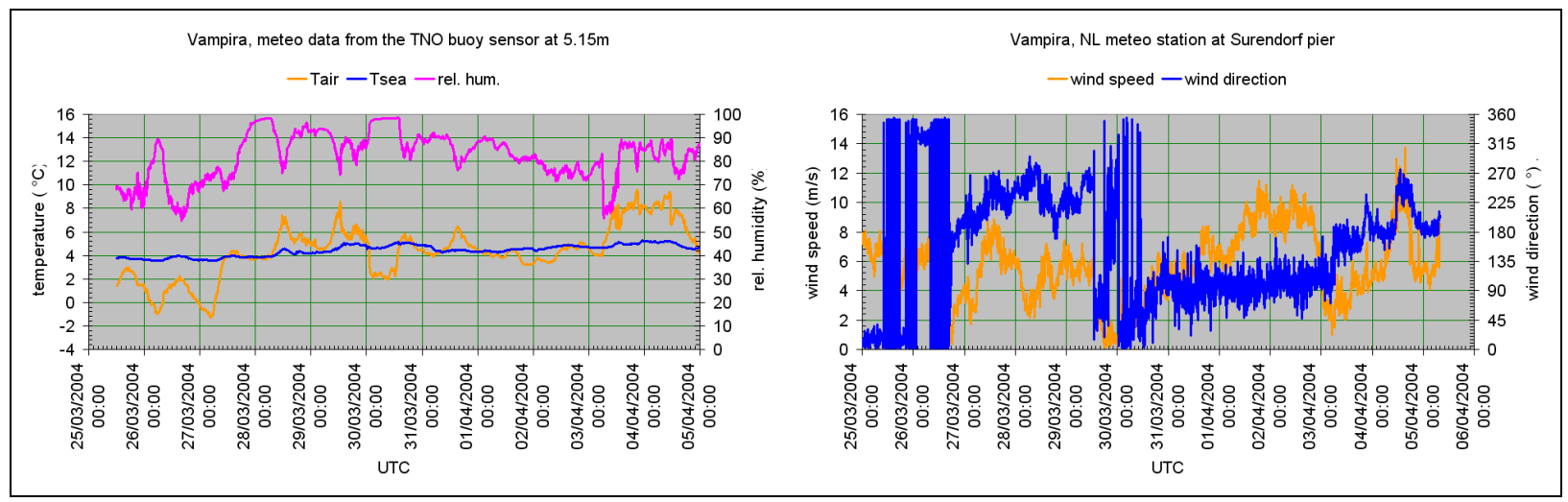

Figure 2: Meteo conditions during the trial period. Left: air temperature, sea temperature and relative humidity measured by the buoy sensor at a height of $5.15 \mathrm{~m}$. Right: wind speed and wind direction from NL meteo station at the pier.

\subsection{Refractive conditions}

In order to obtain insight into the refractive conditions during the trial two methods have been applied. The Canadian method [4] plots the air-to-sea water vapour pressure difference (ASVPD) as a function of the air-to-sea temperature difference (ASTD). Four regions are distinguished:

- region 1: simultaneous RF superrefractive and IR subrefractive conditions. This is the most common situation.

- $\quad$ region 2: simultaneous RF superrefractive and IR superrefractive conditions.

- region 3: simultaneous RF subrefractive and IR superrefractive conditions. This region is the most interested one, because radar may experience a reduced detection range, while IR may experience at the same time an increased detection range.

- $\quad$ region 4: simultaneous RF subrefractive and IR subrefractive conditions.

Figure 3 shows the ASTD-ASVPD relation for the whole trial period using the meteo data from the buoy sensor at 5.15 $\mathrm{m}$. The colours indicate the conditions on the various days. On several days the refractive conditions significantly changed during the day. Most of the time RF superrefractive conditions occurred, while simultaneously IR experienced sub- or superrefraction. But the plot indicates also that there might have been a few occasions along the propagation path with simultaneous RF subrefractive and IR superrefractive conditions.

The French method [5] uses the M-gradient to compute the duct height when $\mathrm{dM} / \mathrm{dh}=0$. When no zero M-gradient is found the $\mathrm{N}$-gradient is used to compute the height where $\mathrm{dN} / \mathrm{dh}=0$. This latter height is called refractive height and is given a negative sign. The method can be applied for both radar and IR/EO. A negative refractive height is not a physical height but an indication for a possible existence of subrefractive conditions.. For the present study the evaporation duct/refractive heights for radar only have been computed by TARMOS [6] and plotted in figure 4. It can be seen that there were time periods on 3 and 4 April 2005 with possibly radar subrefractive conditions. Correlating these with the temperature and relative humidity in figure 2, it is clear that these radar subrefractive conditions occurred when there was simultaneously a high positive ASTD and a moderate relative humidity. Also it became evident that there is a very good match between these periods and the data points in region 3 in the ASTD-ASVPD plot in figure 3.

Both the Canadian and the French method give information on the refractive conditions, but the results are dependent on the measurement height of the meteorological parameters within the duct. The Canadian method can be applied rather easily on board navy vessels and gives almost instantaneously information on the refractive conditions for both radar and IR without using a bulk model. The French method can also be applied on board, but one needs a bulk model to transfer the meteorological parameters to evaporation duct or refractive height.

A third method developed by TNO based upon refractivity gradients is presented in [6].

A more detailed analysis showed that the radar subrefractive conditions might have been met on 3 days covering in total about 4.5 hours out of 225 hours RF measurements. The most interesting day in this respect is 4 April 2005 


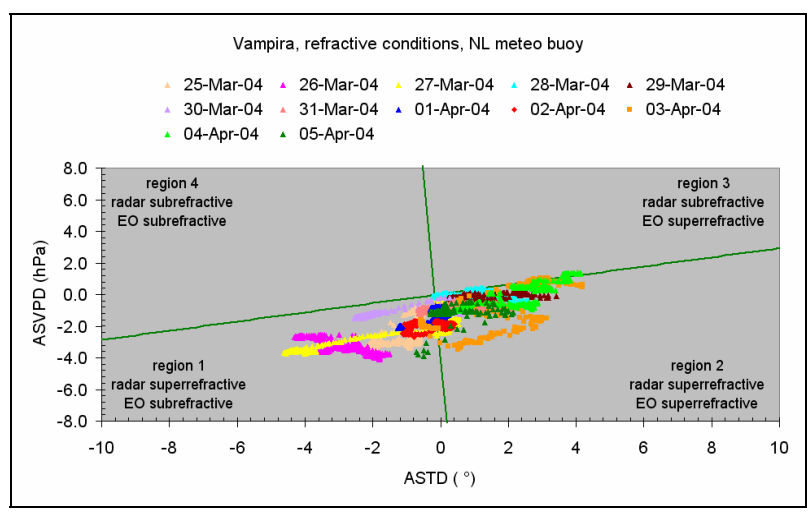

Figure 3: Air-Sea Water Vapour Pressure Difference vs AirSea Temperature Difference for radar and EO propagation during the Vampira trial as suggested by [9] from the sensor on the buoy at a height of $5.15 \mathrm{~m}$.

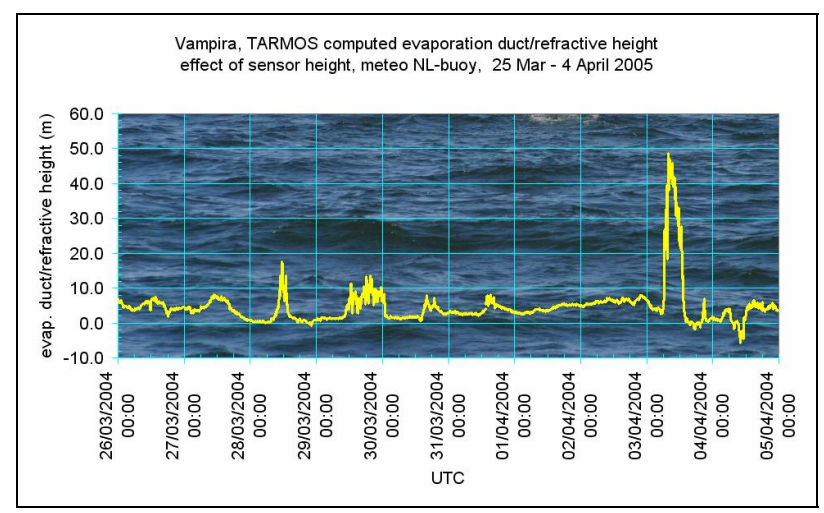

Figure 4: TARMOS computed evaporation duct/refractive height for radar during the Vampira trial as suggested by [10] from the sensor on the buoy at a height of $5.15 \mathrm{~m}$.

\subsection{Effect of height of meteo measurements}

On the buoy meteo sensors were mounted at different heights above the sea surface. These sensors are measuring the air temperature and relative humidity at different positions on the respective vertical profiles. This of course may affect the value of the computed duct height by the TNO model TARMOS [4]. As an example the data from 4 April 2004 have been selected to investigate this.

In figure 5 the air temperature (upper left), relative humidity (upper right) and the resulting duct heights (lower left) obtained from meteo data measured at 2.15, 3.15 and 5.15m above the sea surface are compared. Also the effect on the 1-way propagation factors has been computed in the lower right part of this figure. For comparison the air temperature and relative humidity measured at about $7.5 \mathrm{~m}$ height above 'normal null' at the pier are also given. As mentioned before there is a sudden decrease in air temperature around 11:00 UTC. All buoy sensors and the pier sensor have measured this. This temperature drop coincides with a very quick turn of the wind direction from south towards westsouth-west, meaning that the wind is blowing over the Eckernförder Bucht.

Clearly one can see the increase in temperature (on this day) with height, until about 11:00 UTC where after there is hardly any difference between the temperatures as measured by the considered buoy sensors. In the same time period a decrease of relative humidity is observed with increasing height. The effect on the (computed) duct height is small except for the period between 7:00 ad 11:00 UTC where the computations indicate a 'negative' refractive height. The computed effect on the 1-way path losses as shown in the lower left part of figure 5 is negligible. 


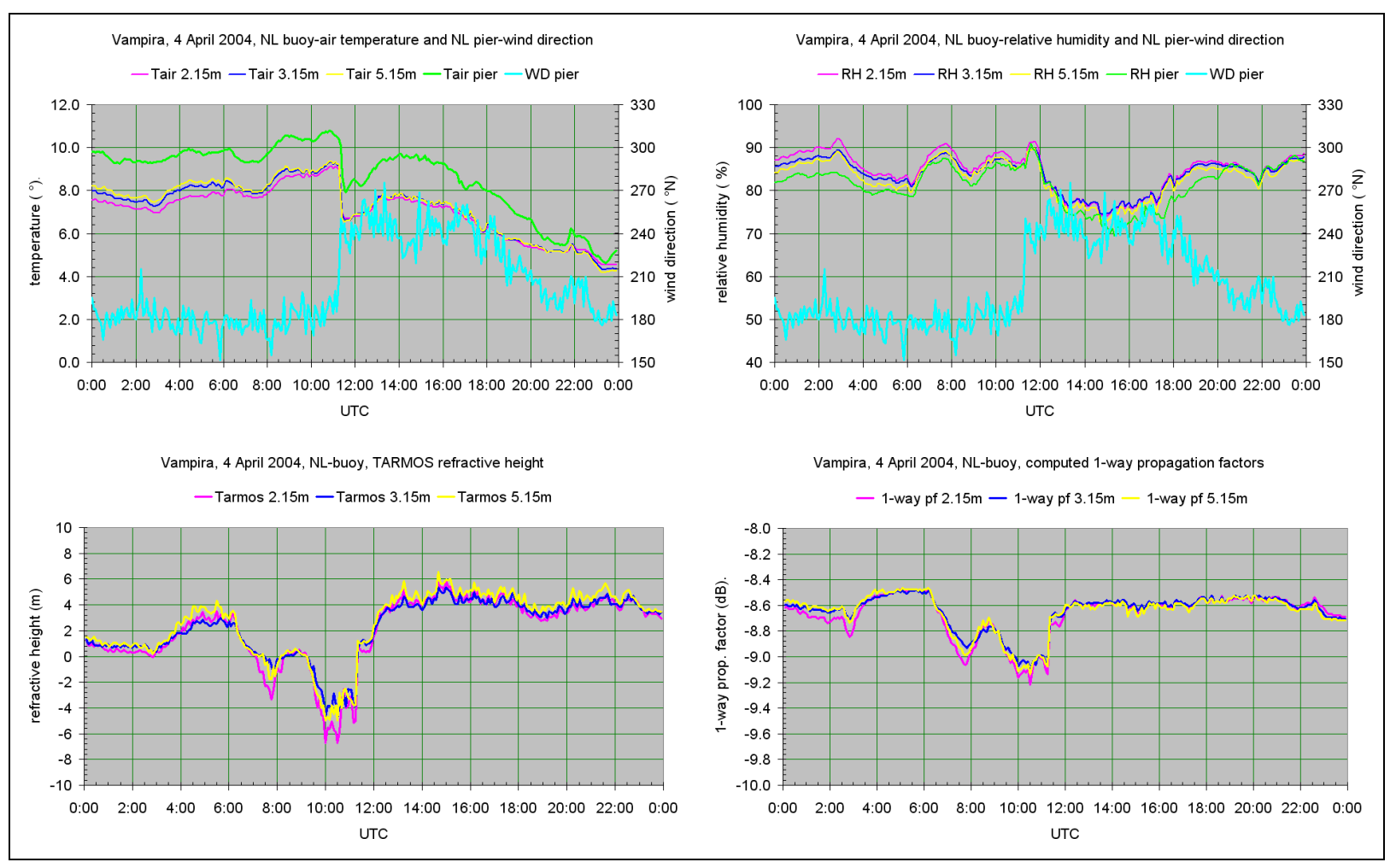

Figure 5: Effect of height of meteo sensors on the computed duct height on 4 April 2004.

\section{RF MEASUREMENTS}

The RF measurements were made from 25 March until 5 April 2004. The received powers at 6 frequencies were averaged over $9 \mathrm{~s}$ and the average was recorded once per minute. The transmitter and receiver antenna covered the whole frequency band from 2-18 GHz and were optically aligned to each other (line of sight). In a later stage however it proved that the transmitter antenna had a vertical offset of $1.7^{\circ}$. Calibration of the link was done by measuring the received power at the 6 frequencies after connecting the output of the transmitter antenna cable to the input of the receiver antenna cable via an appropriate calibrated attenuator. The attenuation value was such that the received powers during calibration were in the same order as the received powers during the actual measurements. By correcting the received powers with the calibration values, the antenna directivity and the free space path loss the RF propagation factors have been obtained. The measured 1-way propagation factors at the 6 frequencies during the entire trial period and the computed evaporation duct/refractive height are given in figure 6. No measured data is available from 3 April 16:25 till 4 April 09:08 UTC due to a electrical power failure.

The dependency of the RF propagation on the evaporation duct height is clearly observed in figure 6 . For the lower frequencies (3.36 and $5.32 \mathrm{GHz}$ ) the measured propagation factor shows about the same behaviour as a function of time with propagation factors up to about $5 \mathrm{~dB}$. The 8.015 and $9.7 \mathrm{GHz}$ are more affected by the atmospheric conditions. Some signal reductions in the order of $5 \mathrm{~dB}$ have been observed during short time periods. However at the same time the signals at 13.45 and $15.71 \mathrm{GHz}$ showed very strong propagation effects. Note that in figure 6 the measured propagation factor for $15.71 \mathrm{GHz}$ is larger than for $13.45 \mathrm{GHz}$. The explanation is the elevation of the transmitter and receiver antenna resulting in a frequency dependent antenna pattern loss. I.e. depending on the frequency, the RF signal is transmitted and received in the mainlobe (lower frequencies) or one of the sidelobes (higher frequencies). 


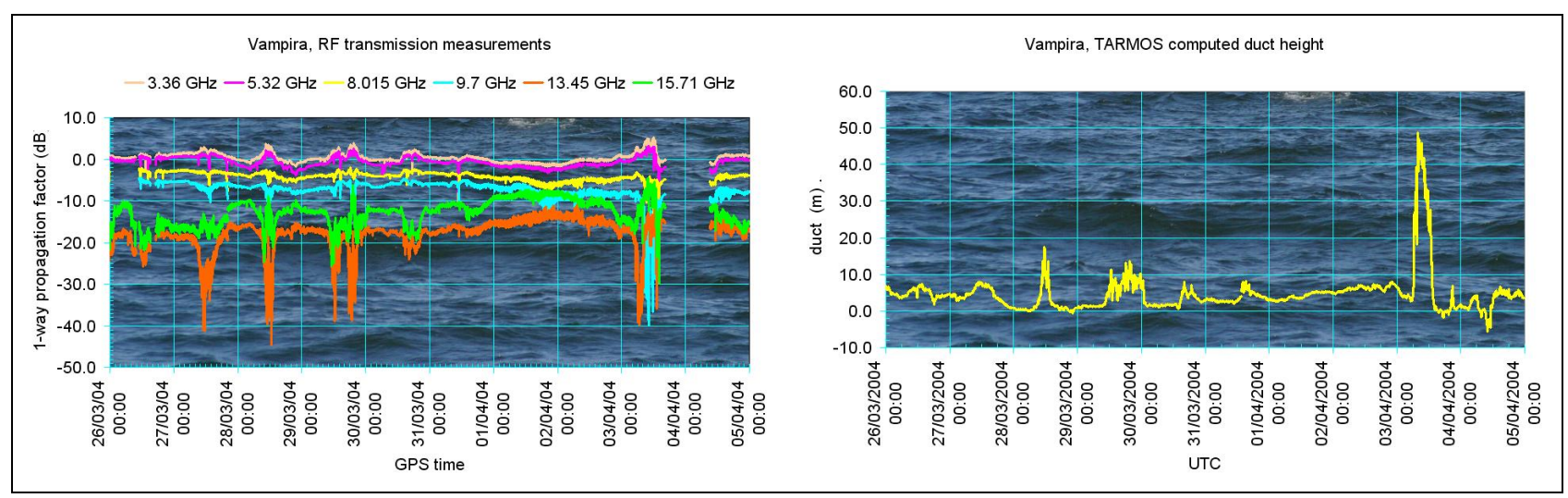

Figure 6: Left: Measured 1-way propagation factors during Vampira at 3.36, 5.32, 8.015, 9.7, 13.45 and 15.71 GHz, path length $8.2 \mathrm{~km}$, transmitter height $11.5 \mathrm{~m}$, receiver height $6.4 \mathrm{~m}$. Right: Duct height computed by TARMOS.

\section{RF PROPAGATION PREDICTION}

\subsection{Model description}

For the validation and study of the propagation effects in dependence of the refractive conditions the TNO model TARMOS [7] and the Signal Science model TERPEM [8] have been used. TARMOS is a research code to predict atmospheric refraction profiles of radar and optical radiation in the marine surface layer. Based on standard meteorological observations of wind speed, air temperature and humidity, and the sea surface temperature, TARMOS computes the vertical profiles of the wind speed, temperature and humidity, turbulent fluxes of momentum, heat and vapour water, the scaling parameters $u^{*}, t^{*}$ and $q^{*}$, the structure function parameter $C_{T}^{2}, C_{Q}^{2}$ and $C_{T Q}$ and other (micro)-meteorological parameters. The results are used to estimate the vertical profiles of the index of refraction $n(z)$, refractivity $N(\mathrm{z})$ and modified refractivity $M(\mathrm{z})$. Currently, TARMOS provides a selection of six different solution methods using different stability functions (Businger-Dyer and Kondo) and different solution methods (based on $C_{D N}$, or Charnock). For the present analysis the Davidson-Kondo model has been used. During the stable conditions bulk models may have a problem to reliably predict the duct height. This is not a mathematical problem of the model but inherent to the atmospheric condition where vertical mixing is limited or absent due to a lack of convection or mechanical mixing.

The TERPEM propagation package is a tool for the forecasting and analysis of refraction, ducting and terrain effects on radio links and radar systems. It is based on state-of-the-art hybrid models combining parabolic equation and ray-trace techniques. Analysis of the output produced by the TERPEM calculations is done by the TGRAPH program. The two programs can be used independently of each other.

\subsection{Case study 28 March 2004}

The first case study concerns a day with almost neutral atmospheric conditions till about 08:00 UTC with a computed duct height of 0-1 m. Stable conditions existed between about 08:00 and 14:00 UTC. In the latter period the ASTD was increasing till about $2^{\circ}$ while the relative humidity decreased from almost $100 \%$ to $75 \%$ at $12: 00$ and increased next to about $95 \%$. This caused the duct height to increase to a maximum computed value of $17 \mathrm{~m}$. The meteorological parameter and computed duct height on this day are presented in figure 7. From the ASTD-ASVPD computations both radar and IR/EO have experienced superrefraction on this day (see figure 3 ). 


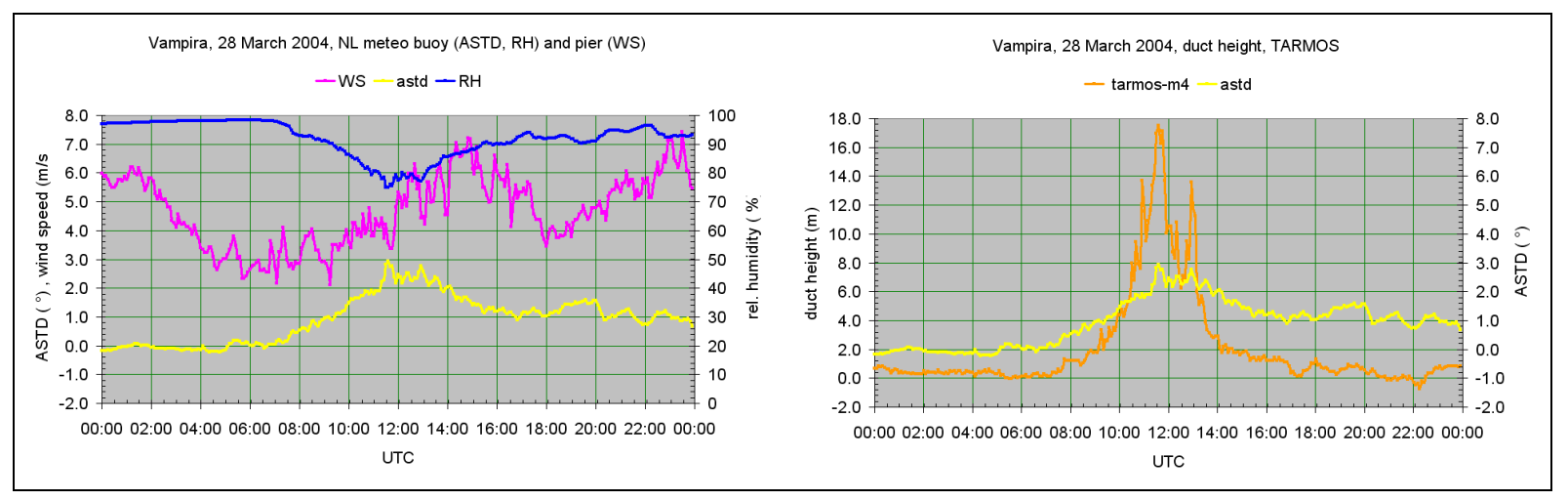

Figure 7: Left: measured relative humidity (buoy) and ASTD (buoy) and wind speed (pier) on 28 March 2004. Right: duct height computed by TARMOS and ASTD (buoy).

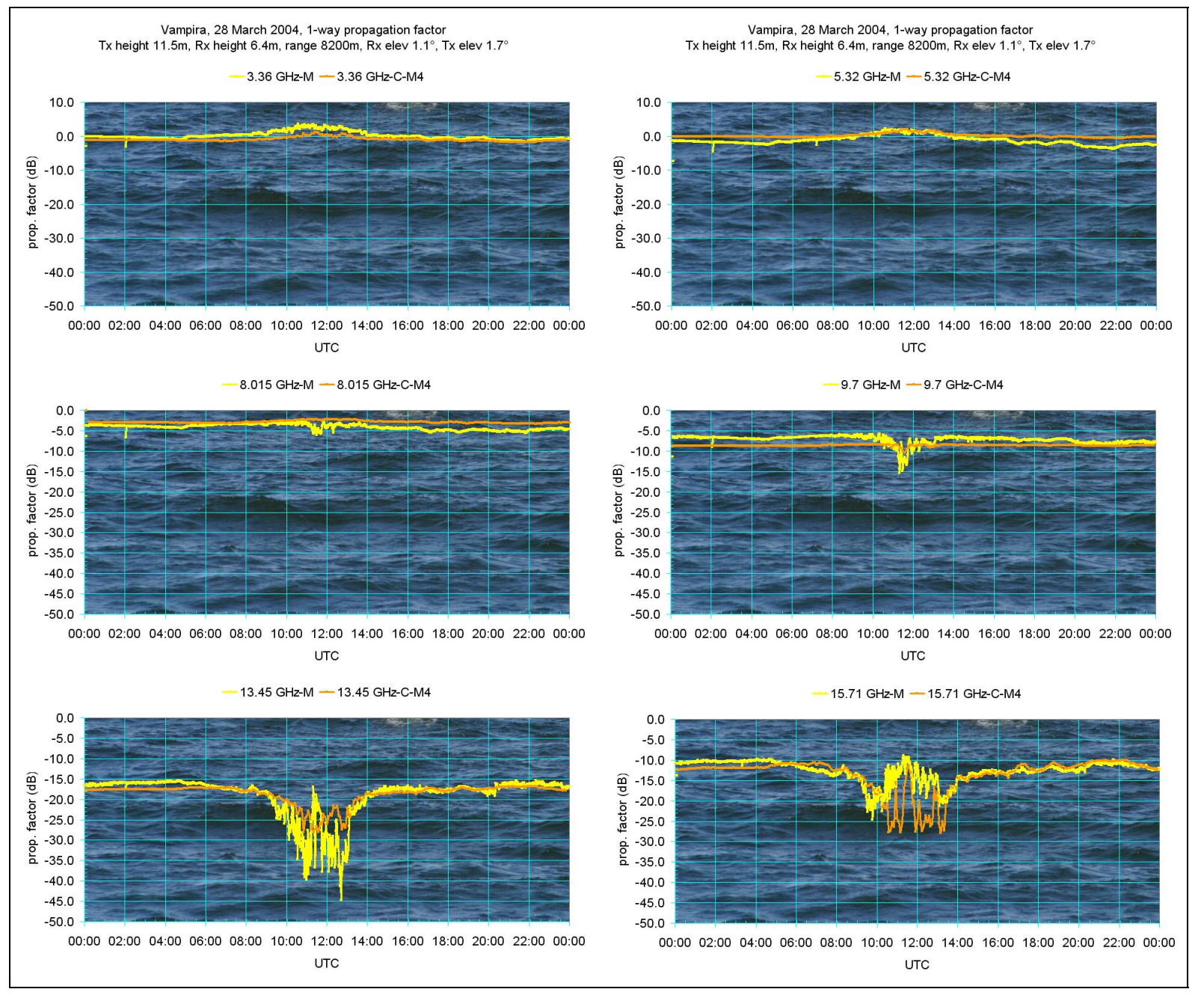

Figure 8: $\quad$ Computed (C-M4) and measured (M) 1-way propagation factors on 28 March 2004 at 3.36, 5.32, 8.015, 9.7, 13.45 and 15.71 GHz, horizontal polarisation. 
The M-profiles computed by TARMOS have been used in TERPEM to compute the 1-way propagation factors for every 5 minutes during the day.

In the computations the actual geometry of the measurements is taken into account i.e. including the elevation of both the transmitter and receiver antenna. The computed and measured 1-way propagation factors for the 6 frequencies are shown in figure 9.

From this it is clear that under unstable and neutral conditions the predictions are in good agreement with the measurements as a function of time. During the period of stable conditions the model computed only a slightly reduced propagation factor at $9.7 \mathrm{GHz}$, while the measurements showed a reduction of about $10 \mathrm{~dB}$. At $8.01 \mathrm{GHz}$ the computations did not show a 'dip' at all. At the higher frequencies the model is in good agreement with the measurements during neutral and unstable atmospheric conditions. The measured decrease of the propagation factor at $13.45 \mathrm{GHz}$ under stable conditions is predicted by the models. At $15.71 \mathrm{GHz}$ the models predict several deep fadings in the period with stable conditions that do not show up in the measurements. This is still to be investigated in more detail.

\subsection{Case study 3 April 2004}

A second case study concerns 3 April 2004. The ASTD-ASVPD diagram computed from the buoy data at $5.15 \mathrm{~m}$ indicates that during this day RF subrefractive conditions were likely to exist between 17:00 and 18:00 UTC. During almost the entire day the ASTD was between $0^{\circ}$ and $4.7^{\circ}$. The meteo data and the computed evaporation duct/refractive height are given in figure 9. Note that only positive values of the refractive height are corresponding to evaporation duct heights. The computed and measured 1-way propagation factors are given in figure 10. From 06:00 till 13:00 UTC there were stable atmospheric conditions. The wind speed was about $3 \mathrm{~m} / \mathrm{s}$ in that period.

All M-profiles computed by TARMOS have been used to compute the propagation factor by TERPEM. Despite the problem that bulk models have to predict reliable duct heights under stable conditions there is a good to very good match between computed and measured propagation factors as a function of time (figure 10), even under stable conditions.

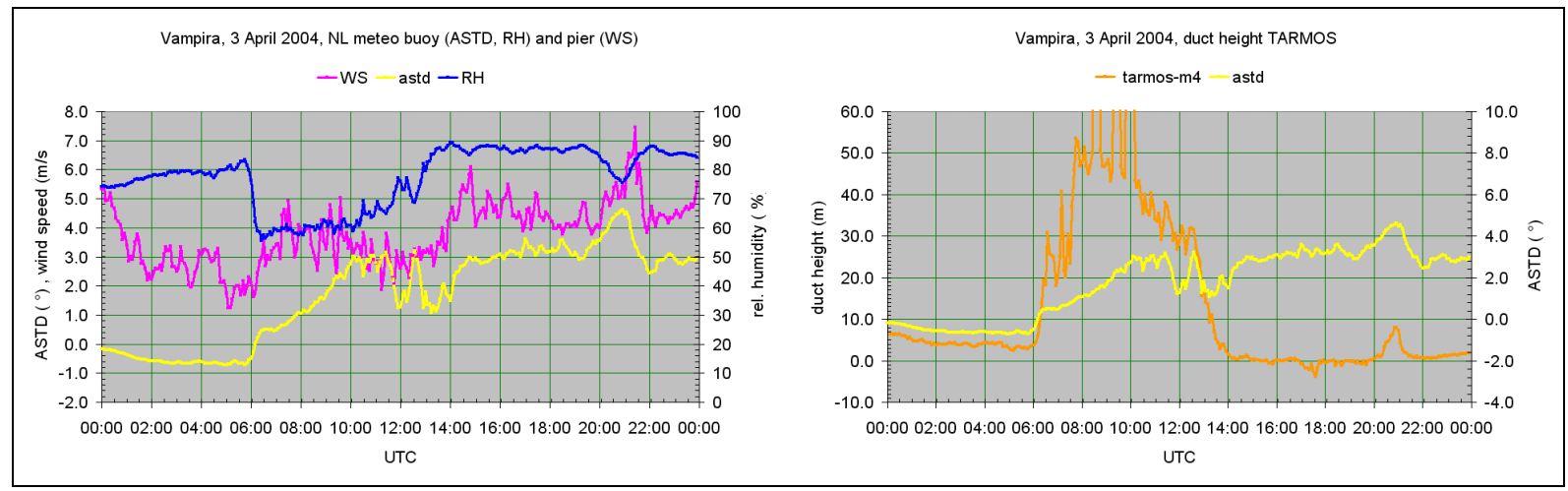

Figure 9: Left: Measured relative humidity (buoy), ASTD (buoy) and wind speed (pier) on 3 April 2004. Right: refractive height computed by TARMOS and ASTD (buoy). 


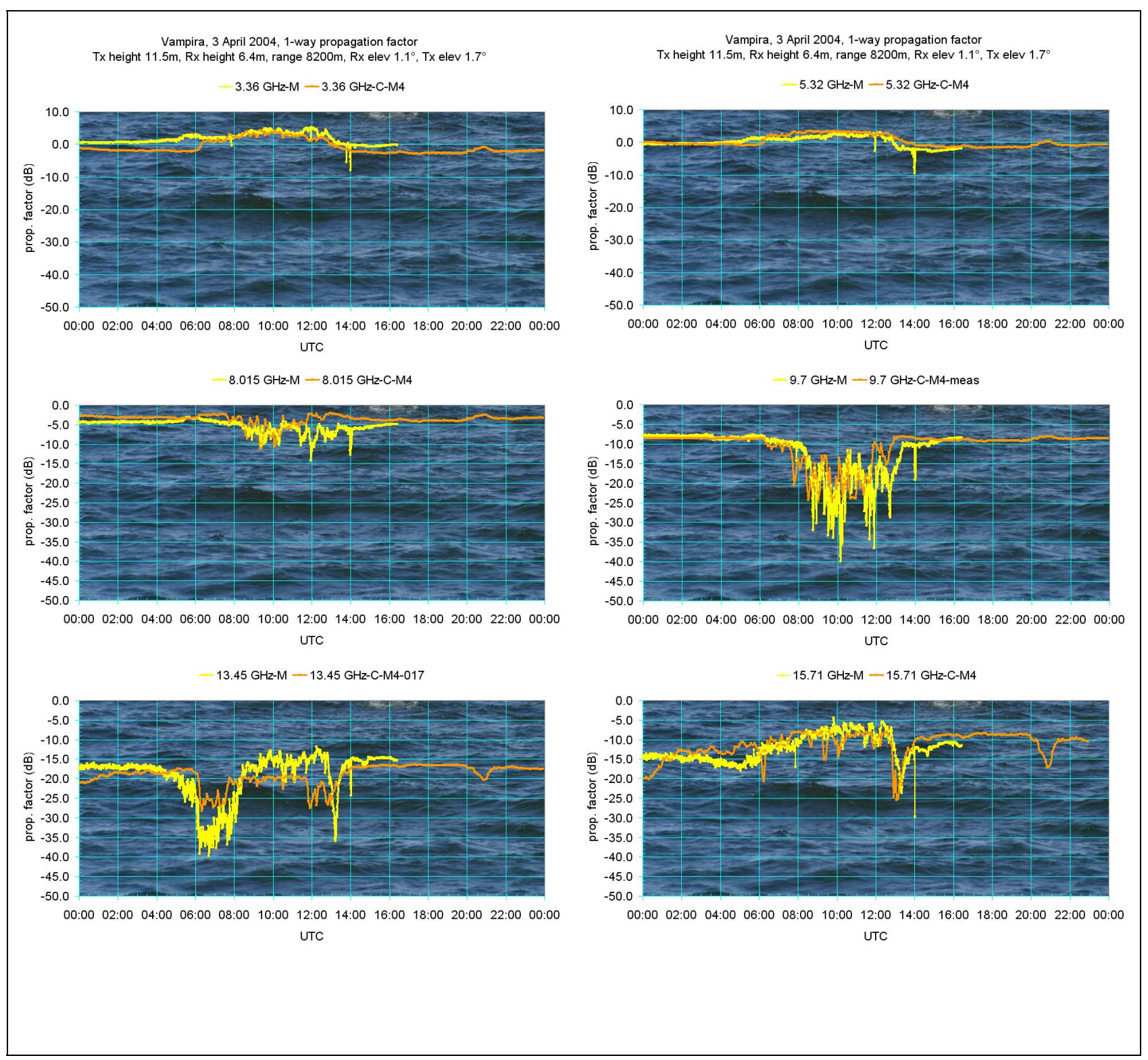

Figure 10: Computed (C-M4) and measured (M) 1-way propagation factors on 3 April 2004 at 3.36, 5.32, 8.015, 9.7, 13.45 and $15.71 \mathrm{GHz}$, horizontal polarisation.

\section{INCIDENCE ANGLE}

The study on radar and infrared/EO synergy with respect to propagation is done using parameters that are common to both radar and EO propagation: transmission loss and angle of arrival or observed elevation position of the RF respectively IR/EO source can be the proper parameters. So far the RF part of the study concentrated on the transmission loss or propagation factor because unfortunately the RF measurements did not include angle-of-arrival measurements. But models can be used to simulate what, in our case, a phase interferometer would have seen under the trial conditions. A study has been started to investigate the phase behaviour of the received radar signal under the various ducting conditions. A special version of TERPEM is used that computes and outputs the complex propagation 
factor. The complex propagation factor is defined as the ratio of the calculated (complex) electromagnetic field $E$ to the (complex) free space field $E_{0}$ and contains the transmission loss and phase.

The phase at two different heights has been used to compute the angle-of-arrival (AOA) of the received RF signal simulating a two element phase interferometer by using

$$
A O A=\operatorname{ASIN}\left(\frac{\lambda \cdot(\varphi \pm k 2 \pi)}{2 \pi d}\right) \quad \mathrm{k}=1,2,3, \ldots . .
$$

where

$\lambda=$ radar wavelength in metres

$\varphi=$ phase difference between the received RF signals at the heights of the interferometer antennas in radians

$\mathrm{d}=$ height difference between the two interferometer antennas in metres

Note that the antennas are considered to be a point receiver. A baseline of $20 \mathrm{~cm}$ has been chosen. Using the computed AOA the height at which the RF source would have been observed under the various atmospheric conditions have been simply computed (free space conditions) by

$H_{\text {observed }}=R^{*} \operatorname{TAN}(A O A)$

where $\mathrm{R}=$ range from $\mathrm{RF}$ source to receiver in metres.

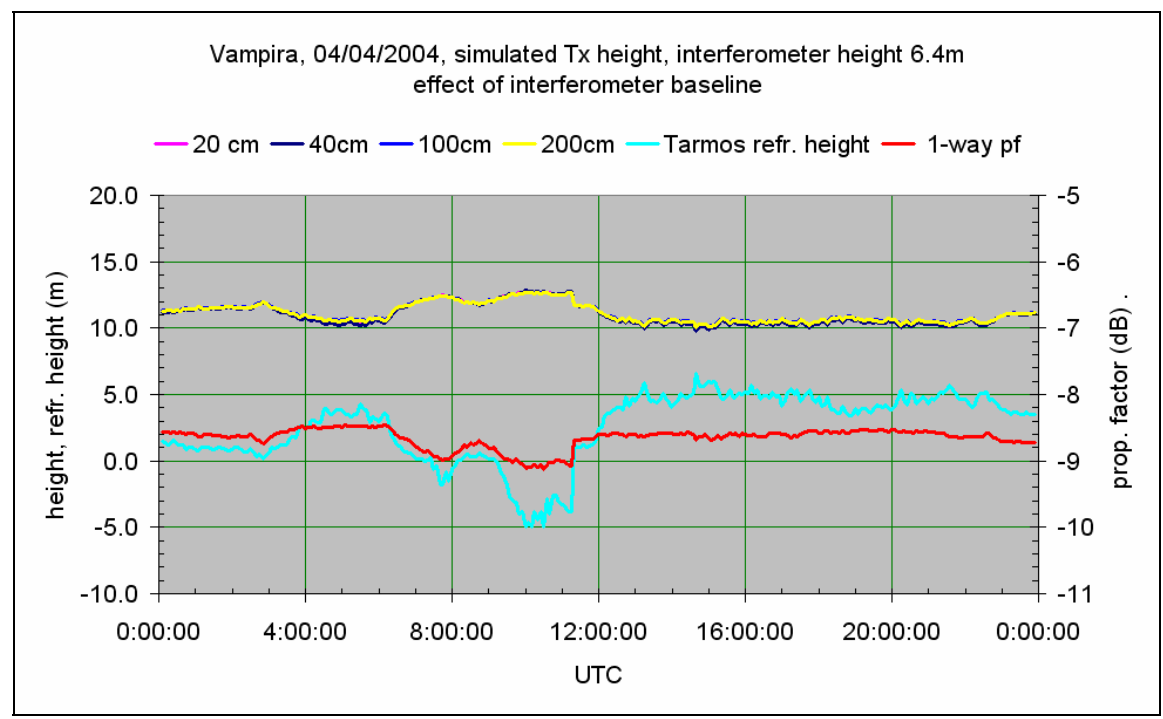

Figure 11: Computed 'observed' height of the RF source, TARMOS refractive height and 1-way propagation factor assuming a two element interferometer at $6.4 \mathrm{~m}$ height, $9.7 \mathrm{GHz}, 8.2 \mathrm{~km}$ range and interferometer baseline of 20, 40, $100 \mathrm{and} 200 \mathrm{~cm}$.

A first result of the computed 'observed' height of the RF source on 4 April 2004 is given in figure 11 for $9.7 \mathrm{GHz}$, a range of $8.2 \mathrm{~km}$ and baselines of 20,40,100 and $200 \mathrm{~cm}$. The figure also shows the TARMOS duct height and the computed 1-way propagation factor.

At first it can be seen that the observed height of the RF source varies under the considered conditions on the $4^{\text {th }}$ April. There is a good correlation with the computed duct height. When the duct height gets lower the observed RF source height increases due to the fact that the AOA becomes more positive at the considered range and interferometer height. Also there are very small differences between the results for the considered baselines. However is must be noted that there may occur substantial differences when the interferometer height is changed and the two antennas are in different propagation lobes. A more detailed study is necessary to fully investigate the behaviour of the AOA under various atmospheric conditions and dependency on interferometer height, range and frequency. These first obtained results are promising. 


\section{SUMMARY AND CONCLUSIONS}

The member nations of NATO AC/323 SET-056/RTG32 have held the propagation experiment VAMPIRA to demonstrate the radar-infrared synergy and/or complementarity. TNO has made amongst other things 1-way RF propagation measurements simultaneously at 6 frequencies from 3-16 GHz once per minute from 25 March-5 April 2004. Simultaneously, meteorological measurements were made by meteo stations ashore at both ends of the path and about halfway by sensors at 5 different heights on a buoy. A complete set of RF and meteo data covering a period of 225 hours has been obtained. A Canadian and French method to obtain insight into the refractive conditions i.e. RF and IR/EO synergy have been applied to the collected meteorological data. The results of both methods agreed very well and showed that there were most of the time RF superrefractive conditions and simultaneously IR sub- and superrefractive conditions along the propagation path. Only during about 4.5 hours RF subrefractive conditions existed.

The effects of evaporation ducting at the propagation at the various frequencies were clearly demonstrated. Some times very deep fadings were present at 13.45 and $15.71 \mathrm{GHz}$ where at the same time almost no effect at $3.36 \mathrm{and} 5.32 \mathrm{GHz}$ was observed. The measured propagation at $15.71 \mathrm{GHz}$ was more enhanced than at $13.45 \mathrm{GHz}$ due to the ducting conditions and the elevation of the transmitter and receiver antenna.

The TNO TARMOS model 4 (Davidson and Kondo) was used to compute the M-profiles, based upon temperature and humidity data from the buoy and wind speed data measured at the pier. The 1-way propagation factors, computed by a combination of TARMOS and TERPEM compared very well to the measured data at all frequencies, although the computed fadings not always were as deep as the measured ones.

First results of computing the angle of arrival of the received RF signal by using the transmission phase are promising and showed an elevation variation of the position of the transmitter antenna due to varying ducting conditions.

Future work will include completing of the computations for the entire measurement period including ducting effects on the angle-of-arrival, correlating the predictions and measurements, comparing the RF data with the IR transmission data that were simultaneously measured along the same path and classifying the results to combined RF-IR refractive conditions.

\section{ACKNOWLEDGEMENT}

I like to express my appreciation to the members of AC/323 SET-056/RTG32 that have participated to the VAMPIRA trial, the WTD71 for hosting the trial and providing the facilities, ships and support during the trial.

\section{REFERENCES}

[1] H.J.M. Heemskerk, "Vampira Radar and Infrared Propagation Synergism Trial", Eur. Conference on Propagation and Systems, 15-18 March 2005, Brest France.

[2] H.J.M. Heemskerk, "VAMPIRA RF Propagation measurement and model validation", Eur. Conference on Propagation and Systems, 15-18 March 2005, Brest France.

[3] J. Foerster and J. Riechen, "In-situ measurements of marine boundary layer refractive variability during the Vampira experiment", Eur. Conference on Propagation and Systems, 15-18 March 2005, Brest France.

[4] L. Forand "A Study into Infrared Search and Track \& Radar Synergy», DRDC Valcartier TR 2003-044, June 2003.

[5 J. Claverie, and Y. Hurtaud, "Refractive height", presented to the AC/323 SET056/RTG32, Nov 2004

[6] G.J. Kunz, H.J.M. Heemskerk, L. van Eijk, "Comparison of atmospheric refraction at radar and optical wavelengths", SPIE Europe International Symposium on Remote Sensing 2005, 19-22 Sep 2005, Bruges Belgium.

[7] G. J. Kunz, "A bulk model to predict optical turbulence in the marine surface layer", TNO report FEL-96-A053, April 1996.

[8] M. F. Levy and K. H Craig, "Millimetre-wave propagation in the evaporation duct", Agard Conference proceedings no. 454, pp. 26-1; 26-10, Copenhagen, Denmark, 9-13 October 1993. 